\title{
THE RELATION BETWEEN DASHES AND FLARES (PHYSICAL NATURE OF THE DASH PHENOMENA)
}

\author{
D. A. KUZNETSOV \\ Siberian Institute of Terrestrial Magnetism, \\ Ionosphere and Radiowave Propagation, Academy of Sciences, Irkutsk, U.S.S.R. \\ and \\ A. A. SHPITALNAYA \\ The Pulkovo Observatory, Leningrad, U.S.S.R.
}

\begin{abstract}
The phenomenon of dashes in the eruptive loop prominences is found to represent the part of the solar flare process which includes all the.levels of the Sun's atmosphere. For $75 \%$ of observations a coincidence of dashes and flares in space and time was discovered. The rest of the dash observations may be interpreted as the excitation of dashes by the flares arising outside the limb.

Following the solar flare theory of Alfvén and Carlquist, the possibility to interpret the dashes as the eruptive instability of the pinch-effect is discussed. The computation of flare-current magnetic field gives the value $H \sim 3 \cdot 10^{4}$ oersted, its order being in agreement with the observed value.
\end{abstract}

1. Disturbed short-lived $\left(1-25^{\mathrm{m}}\right)$ small features, the so-called 'dashes', are observed at a height range of $10000 \mathrm{~km}$ to $70000 \mathrm{~km}$ above the solar limb (Young, 1895; Ellison, 1959; Billings, 1957; Prokofjeva, 1957; Shpitalnaya, 1964; Krat, 1968). The dashes arise in the legs of arches of loop eruptive prominences (Ellison, 1959; Billings, 1957). One of the present authors has recently discovered that dashes possess strong distinctly localized magnetic fields (Shpitalnaya and Vjalshin, 1970).

Magnetic field strengths as high as 10000 oersted were measured on spectrograms of $\mathrm{D}_{3}$ and $\mathrm{H} \beta$ lines taken with a Wollaston prism and a $\frac{1}{4} \lambda$ plate attached to the Pulkova coronal spectrograph.

An asymmetric line-of-sight velocity structure characteristic of dashes was observed in spectra obtained in the region of the strong $\mathrm{H} \alpha, \mathrm{H} \beta, \mathrm{He} \mathrm{D}_{3}, \mathrm{Na} \mathrm{D}_{1} \mathrm{D}_{2}$, etc. lines (Young, 1895; Ellison, 1959; Billings, 1957; Prokofjeva, 1957; Shpitalnaya, 1964; Krat, 1968). The velocities of the dashes are within the limits of $25-500 \mathrm{~km} / \mathrm{s}$. Plasma outflow from the dashes activates the surrounding corona. The density of the latter is increased (Kleczek and Hansen, 1962; Shpitalnaya, 1964).

The spectrograms of $D_{3}$ taken at Pulkovo on August 19, 1964 are shown in Figures 1a and $1 \mathrm{~b}$. During a period of less than one minute the plasma outflow velocity increased from $107 \mathrm{~km} / \mathrm{s}$ to $200 \mathrm{~km} / \mathrm{s}$, i.e. the plasma was ejected with an acceleration of about $2 \mathrm{~km} / \mathrm{s}^{2}$, which is higher than the acceleration of gravity.

2. It is usually supposed that flares activate prominences if the angular distance between them does not exceed $20^{\circ}$ and if dashes originate during the existence of the flare. A detailed comparison was made between the location and the time of development of these non-steady phenomena. The flare data and coordinates are given in Solar 


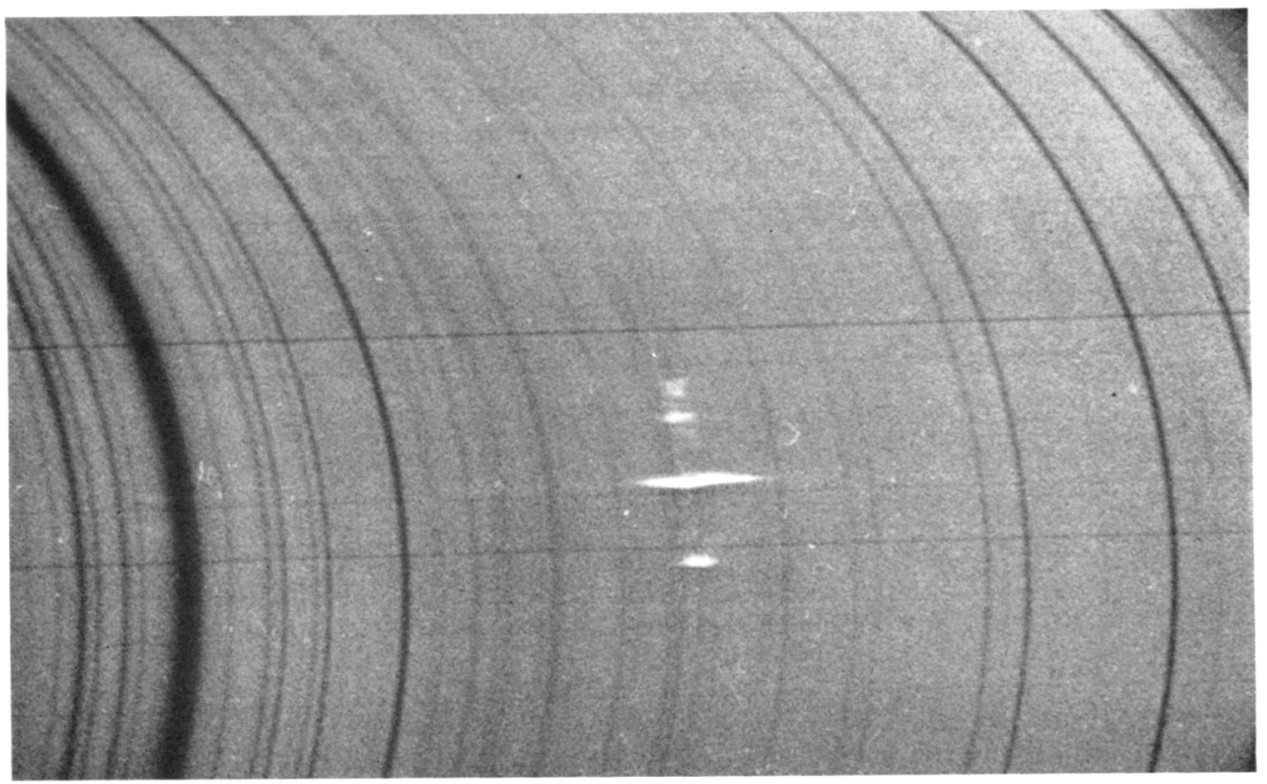

(a)

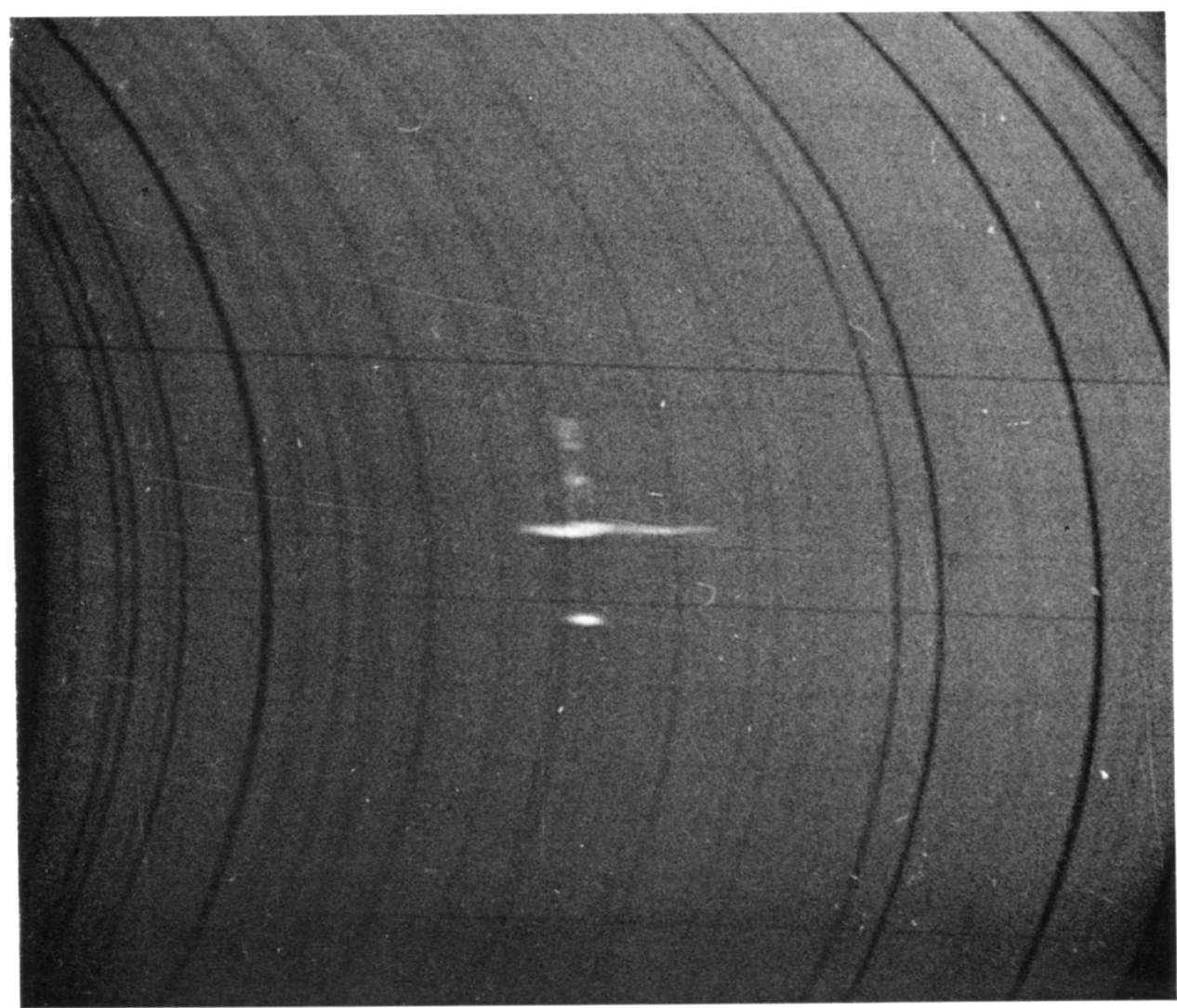

(b)

Fig. 1. He-D limb spectra obtained at Pulkovo observatory on August 19, 1964; the height above the solar limb was about $12^{\prime \prime}$. (a) $13^{\mathrm{h}} 10^{\mathrm{m}}$ UT the maximum line-of-sight velocity of the dash $\left(V_{\max }\right)$ is about $100 \mathrm{~km} / \mathrm{s}$; (b) $13^{\mathrm{h}} 11^{\mathrm{m}} \mathrm{UT}, V_{\max }=200 \mathrm{~km} / \mathrm{s}$. The acceleration of plasma ejection was about $2 \mathrm{~km} / \mathrm{s}^{2}$. 
TABLE I

Illustration of the connection between dashes and flare activity

\begin{tabular}{lrrr}
\hline Date & $\begin{array}{l}\text { Number } \\
\text { of dash events }\end{array}$ & $\begin{array}{l}\text { Number of coincident } \\
\text { flare events }\end{array}$ & $\begin{array}{l}\text { Percentage } \\
\text { of coincidence }\end{array}$ \\
\hline 1960 & 15 & 14 & $93 \%$ \\
1961 & 3 & 3 & $100 \%$ \\
1962 & 11 & 8 & $73 \%$ \\
1963 & 7 & 5 & $71 \%$ \\
1964 & 4 & 2 & $50 \%$ \\
1965 & 4 & 2 & $50 \%$ \\
1966 & 16 & 11 & $69 \%$ \\
\hline & 60 & 45 & mean \\
\hline
\end{tabular}

Data, Zurich Quarterly Bulletins, Fraunhofer Institute Maps, and observations obtained at Pulkovo. The results are summarized in Table I.

It follows that some of the dashes may be associated with flares on the invisible solar hemisphere (Westin and Liszka, 1970).

3. This fact does not raise doubts that dashes are part of a complex flare process. According to the Alfven and Carlquist mechanism (1967) the origin of a flare is due to an electric circuit breakdown in a vertical current, $I \sim 10^{11}-10^{12} \mathrm{~A}$, passing through an area of the photosphere with a diameter of $\sim 10^{9} \mathrm{~cm}$, and is accompanied by the generation of an oscillatory voltage of about $10^{9} \mathrm{~V}$. It is proposed that dashes are formed as a consequence of the so-called 'eruptive instability' of a pinch (Arzimovich, 1961). Plasmoids are ejected from the surface of the pinch with velocities close to those of plasma compression. The latter can be as high as several hundred kilometers per second in laboratory experiments (Arzimovich, 1961). The total energy applied to the plasma during one discharge cycle, $\left(t_{2}-t_{1}\right)$ must be higher than all the forces acting in the plasma and the radiative loss. As $m_{i} \gg m_{e}$ the power applied to a pinch of unit length may be expressed as

$$
\int_{t_{1}}^{t_{2}} W d t \gtrsim \int_{t_{1}}^{t_{2}} k N_{i}(t) T_{i}(t) \mathrm{d} t+\frac{1}{2} M V^{2}+\frac{1}{8 \pi} \int_{r_{2}}^{r_{1}}\left(H^{2}+H_{c}^{2}\right) r d r,
$$

where $W$ is the power, $r$ is the radius of the pinch, $N$ is the concentration, $T$ is the temperature, $k$ is the Boltzmann constant, $H$ is the magnetic field of the current, $H_{c}$ is the 'catching' magnetic field, $M$ is the pinch mass, and $V$ is the return velocity. From this relation the magnetic field strengths compressing the plasma may be obtained. It is assumed that the time for magnetic field penetration into the plasma is much greater than the period of the oscillation of the flare instability (Alfven and Carlquist, 1967). It is also assumed that the velocity of the plasmoids ejected from the pinch is equal to that of the compression of the pinch. 
Omitting intermediate calculations we find for the mean magnetic field strength $\bar{H}$ :

$$
\bar{H} \approx\left[\frac{16 \pi b I U_{0}}{\bar{V}\left(r_{1}+r_{2}\right)}\right]^{1 / 2}
$$

where $\mathbf{U}_{0}$ is the initial voltage value, $\bar{V}$ is the mean velocity of eruptive motions, $b$ is the factor of proportionality.

The total flare current branches in a large number of channels in the corona. Each of these channels is a current circuit from prominence knots.

We consider that there are at least $10^{3}$ separate channels representing circuits from 10 dashes. This is in accordance with the observed fine structure of prominences. If the initial radius of a pinch is $r_{1} \sim 10^{9} \mathrm{~cm}, \bar{V} \sim 10^{7} \mathrm{~cm} / \mathrm{s}, I \sim 10^{11} \mathrm{~A}$, and the voltage is $10^{9} \mathrm{~V}$ (Severny, 1965; Alfvén and Carlquist, 1967) then

$$
H \sim 3 \cdot 10^{4} \mathrm{Oe} .
$$

This estimate of the magnetic field strength coincides with the data within an order of magnitude (Shpitalnaya and Vjalshin, 1970).

This discrepancy may be due to the fact that effects distorting the measured line profiles and masked Zeeman line splitting are present in these observations as this investigation indicates a lower limit of $\bar{H}$ in the dashes.

We note that fields with strength $10^{4} \mathrm{Oe}$ are necessary to explain the observed metal line splitting in flares (Alikaeva, 1969).

\section{References}

Alfvén, H. and Carlquist, P.: 1967, Solar Phys. 1, 220.

Alikaeva, K. V.: 1969, Astron. Astrophys. 8, 92.

Arzimovich, L. A.: 1961, Ruled Thermonuclear Reactions, Moscow.

Billings, D. D.: 1957, Publ. Astron. Soc. Pacific 69, 407.

Ellison, M. A.: 1959, The Sun and Its Influence, London.

Kleczek, J. and Hansen, R. T.: 1962, Publ. Astron. Soc. Pacific 74, 441.

Krat, V.: 1968, in Y. Öhman (ed.), 'Mass Motions in Solar Flares and Related Phenomena', Nobel Symp. 9, 93.

Prokofijeva, I. A.: 1957, Solar-Geophysical Data 8, ESSA Boulder.

Severny, A. B.: 1965, Astron. J. 42, 217.

Shpitalnaya, A. A.: 1964, Publ. Pulkovo Obs. 177, 60.

Shpitalnaya, A. A. and Vjalshin, G. F.: 1970, Solar-Geophysical Data, 4, ESSA Boulder.

Westin, H. and Liszka, L.: 1970, Solar Phys. $2,3$.

Young, H. A.: 1895, The Sun, London.

\section{Discussion}

Kuperus: Could you give a reference of the experimental results mentioned in your paper on the excitation of plasmoids by the interruption of a current?

Soboleva: Arzimovich, L. A.: 1961, Controlled Thermonuclear Reactions, Moscow. 